Article

\title{
Generalizations of Hardy Type Inequalities by Abel-Gontscharoff's Interpolating Polynomial
}

\author{
Kristina Krulić Himmelreich ${ }^{1}$, Josip Pečarić ${ }^{2}$, Dora Pokaz ${ }^{3, *}$ and Marjan Praljak ${ }^{4}$ \\ 1 Faculty of Textile Technology, University of Zagreb, Prilaz Baruna Filipovica 28a, 10000 Zagreb, Croatia; \\ kkrulic@ttf.hr \\ 2 Croatian Academy of Sciences and Arts, Trg Nikole Šubića Zrinskog, 10000 Zagreb, Croatia; \\ pecaric@element.hr \\ 3 Faculty of Civil Engineering, University of Zagreb, Fra Andrije Kačića-Miošića 26, 10000 Zagreb, Croatia \\ 4 Faculty of Food Technology and Biotechnology, University of Zagreb, 6 Pierottijeva Street in Zagreb, \\ 10000 Zagreb, Croatia; marjan.praljak@pbf.unizg.hr \\ * Correspondence: dora@grad.hr or dora.pokaz@grad.unizg.hr
}

check for

updates

Citation: Krulić Himmelreich, K.; Pečarić, J.; Pokaz, D.; Praljak, M. Generalizations of Hardy Type Inequalities by Abel-Gontscharoff's Interpolating Polynomial. Mathematics 2021, 9, 1724. https:// doi.org/10.3390/math9151724

Academic Editor: Valery Karachik

Received: 9 June 2021

Accepted: 19 July 2021

Published: 22 July 2021

Corrected: 27 March 2023

Publisher's Note: MDPI stays neutral with regard to jurisdictional claims in published maps and institutional affiliations.

Copyright: (c) 2021 by the authors. Licensee MDPI, Basel, Switzerland. This article is an open access article distributed under the terms and conditions of the Creative Commons Attribution (CC BY) license (https:/ / creativecommons.org/licenses/by/ $4.0 /)$.
Abstract: In this paper, we extend Hardy's type inequalities to convex functions of higher order. Upper bounds for the generalized Hardy's inequality are given with some applications.

Keywords: inequalities; Hardy type inequalities; Abel-Gontscharoff interpolating polynomial; Green function; Chebyshev functional; Grüss type inequalities; Ostrowski type inequalities; convex function; kernel; upper bounds

\section{Introduction and Preliminaries}

Let $\left(\Sigma_{1}, \Omega_{1}, \mu_{1}\right)$ and $\left(\Sigma_{2}, \Omega_{2}, \mu_{2}\right)$ be measure spaces with positive $\sigma$-finite measures. For a measurable function $f: \Omega_{2} \rightarrow \mathbb{R}$, let $A_{k}$ denote the linear operator

$$
A_{k} f(x):=\frac{1}{K(x)} \int_{\Omega_{2}} k(x, t) f(t) d \mu_{2}(t),
$$

where $k: \Omega_{1} \times \Omega_{2} \rightarrow \mathbb{R}$ is measurable and non-negative kernel with

$$
0<K(x):=\int_{\Omega_{2}} k(x, t) d \mu_{2}(t), \quad x \in \Omega_{1}
$$

The following result was given in [1] (see also [2]), where $u$ is a positive function on $\Omega_{1}$.

Theorem 1. Let $u$ be a weight function, $k(x, y) \geq 0$. Assume that $\frac{k(x, y)}{K(x)} u(x)$ is locally integrable on $\Omega_{1}$ for each fixed $y \in \Omega_{2}$. Define $v$ by

$$
v(y):=\int_{\Omega_{1}} \frac{k(x, y)}{K(x)} u(x) d \mu_{1}(x)<\infty .
$$

If $\phi$ is a convex function on the interval $I \subseteq \mathbb{R}$, then the inequality

$$
\int_{\Omega_{1}} \phi\left(A_{k} f(x)\right) u(x) d \mu_{1}(x) \leq \int_{\Omega_{2}} \phi(f(y)) v(y) d \mu_{2}(y)
$$

holds for all measurable functions $f: \Omega_{2} \rightarrow \mathbb{R}$, such that $\operatorname{Im} f \subseteq I$, where $A_{k}$ is defined by (1) and (2). 
Inequality (4) is generalization of Hardy's inequality. G. H. Hardy [3] stated and proved that the inequality

$$
\int_{0}^{\infty}\left(\frac{1}{x} \int_{0}^{x} f(t) d t\right)^{p} d x \leq\left(\frac{p}{p-1}\right)^{p} \int_{0}^{\infty} f^{p}(x) d x, p>1
$$

holds for all $f$ non-negative functions such that $f \in L^{p}\left(\mathbb{R}_{+}\right)$and $\mathbb{R}_{+}=(0, \infty)$. The constant $\left(\frac{p}{p-1}\right)^{p}$ is sharp. More details about Hardy's inequality can be found in $[4,5]$.

Inequality (5) can be interpreted as the Hardy operator $H: H f(x):=\frac{1}{x} \int_{0}^{x} f(t) d t$, maps $L^{p}$ into $L^{p}$ with the operator norm $p^{\prime}=\frac{p}{p-1}$.

In this paper, we consider the difference of both sides of the generalized Hardy's inequality

$$
\int_{\Omega_{2}} \phi(f(y)) v(y) d \mu_{2}(y)-\int_{\Omega_{1}} \phi\left(A_{k} f(x)\right) u(x) d \mu_{1}(x)
$$

and obtain new inequalities that hold for $n$-convex functions.

Now, we recall $n$-convex functions. There are two parallel notations. First, is given by E. Hopf in 1926 and second by T. Popoviciu in 1934. E. Hopf defined that the function $f$ is $n$-convex if difference $\left[x_{0}, \ldots, x_{n+1}, f\right]$ is nonnegative. The ordinary convex function is 1-convex. For more details see [6]. In the second definition $f:[\alpha, \beta] \rightarrow \mathbb{R}$ is $n$-convex $n \geq 0$, if its $n$-th order divided differences $\left[x_{0}, \ldots, x_{n} ; f\right]$ are nonnegative for all choices of $(n+1)$ distinct points $x_{i} \in[\alpha, \beta]$. By second definition 0 -convex function is nonnegative, 1 -convex function is non-decreasing and 2-convex function is convex in the usual sense. If an $n$-convex function is $n$ times differentiable, then $\phi^{(n)} \geq 0$. (see [7]).

An important role in the paper will be played by Abel-Gontscharoff interpolation, which was first studied by Whittaker [8], and later by Gontscharoff [9] and Davis [10]. The Abel-Gontscharoff interpolation for two points and the remainder in the integral form is given in the following theorem (for more details see [11]).

Theorem 2. Let $n, m \in \mathbb{N}, n \geq 2,0 \leq m \leq n-1$ and $\phi \in C^{n}([\alpha, \beta])$. Then

$$
\phi(u)=Q_{n-1}(\alpha, \beta, \phi, u)+R(\phi, u),
$$

where $Q_{n-1}$ is the Abel-Gontscharoff interpolating polynomial for two-points of degree $n-1$, i.e.,

$$
\begin{aligned}
& Q_{n-1}(\alpha, \beta, \phi, u)=\sum_{s=0}^{m} \frac{(u-\alpha)^{s}}{s !} \phi^{(s)}(\alpha) \\
& +\sum_{r=0}^{n-m-2}\left[\sum_{s=0}^{r} \frac{(u-\alpha)^{m+1+s}(\alpha-\beta)^{r-s}}{(m+1+s) !(r-s) !}\right] \phi^{(m+1+r)}(\beta)
\end{aligned}
$$

and the remainder is given by

$$
R(\phi, u)=\int_{\alpha}^{\beta} G_{m n}(u, t) \phi^{(n)}(t) d t
$$

where $G_{m n}(u, t)$ is Green's function defined by

$$
G_{m n}(u, t)=\frac{1}{(n-1) !} \begin{cases}\sum_{s=0}^{m}\left(\begin{array}{c}
n-1 \\
s
\end{array}\right)(u-\alpha)^{s}(\alpha-t)^{n-s-1}, & \alpha \leq t \leq u \\
-\sum_{s=m+1}^{n-1}\left(\begin{array}{c}
n-1 \\
s
\end{array}\right)(u-\alpha)^{s}(\alpha-t)^{n-s-1}, & u \leq t \leq \beta\end{cases}
$$


Remark 1. For $\alpha \leq t, u \leq \beta$ the following inequalities hold

$$
\begin{aligned}
&(-1)^{n-m-1} \frac{\partial^{s} G_{m n}(u, t)}{\partial u^{s}} \geq 0, \quad 0 \leq s \leq m \\
&(-1)^{n-s} \frac{\partial^{s} G_{m n}(u, t)}{\partial u^{s}} \geq 0, \quad m+1 \leq s \leq n-1 .
\end{aligned}
$$

\section{Generalizations of Hardy's Inequality}

Our first result is an identity related to generalized Hardy's inequality. We apply interpolation by the Abel-Gontscharoff polynomial and get the following result.

Theorem 3. Let $\left(\Sigma_{1}, \Omega_{1}, \mu_{1}\right)$ and $\left(\Sigma_{2}, \Omega_{2}, \mu_{2}\right)$ be measure spaces with positive $\sigma$-finite measures. Let $u: \Omega_{1} \rightarrow \mathbb{R}$, be a weight function and $v$ is defined by (3). Let $A_{k} f(x), K(x)$ be defined by (1) and (2) respectively, for a measurable function $f: \Omega_{2} \rightarrow[\alpha, \beta]$ and let $n, m \in \mathbb{N}, n \geq 2$, $0 \leq m \leq n-1, \phi \in C^{n}([\alpha, \beta])$ and $G_{m n}$ be defined by (6). Then

$$
\begin{aligned}
& \int_{\Omega_{2}} \phi(f(y)) v(y) d \mu_{2}(y)-\int_{\Omega_{1}} \phi\left(A_{k} f(x)\right) u(x) d \mu_{1}(x) \\
& =\sum_{s=1}^{m} \frac{\phi^{(s)}(\alpha)}{s !}\left(\int_{\Omega_{2}}(f(y)-\alpha)^{s} v(y) d \mu_{2}(y)-\int_{\Omega_{1}}\left(A_{k} f(x)-\alpha\right)^{s} u(x) d \mu_{1}(x)\right) \\
& +\sum_{r=0}^{n-m-2} \sum_{s=0}^{r} \frac{(-1)^{r-s}(\beta-\alpha)^{r-s} \phi^{(m+1+r)}(\beta)}{(m+1+s) !(r-s) !}\left(\int_{\Omega_{2}}(f(y)-\alpha)^{m+1+s} v(y) d \mu_{2}(y)\right. \\
& \left.\quad-\int_{\Omega_{1}}\left(A_{k} f(x)-\alpha\right)^{m+1+s} u(x) d \mu_{1}(x)\right) \\
& +\int_{\alpha}^{\beta}\left(\int_{\Omega_{2}} G_{m n}(f(y), t) v(y) d \mu_{2}(y)-\int_{\Omega_{1}} G_{m n}\left(A_{k} f(x), t\right) u(x) d \mu_{1}(x)\right) \phi^{(n)}(t) d t .
\end{aligned}
$$

Proof. Using Theorem 2 we can represent every function $\phi \in C^{n}([\alpha, \beta])$ in the form

$$
\begin{aligned}
\phi(u) & =\sum_{s=0}^{m} \frac{(u-\alpha)^{s}}{s !} \phi^{(s)}(\alpha) \\
& +\sum_{r=0}^{n-m-2}\left[\sum_{s=0}^{r} \frac{(u-\alpha)^{m+1+s}(-1)^{r-s}(\beta-\alpha)^{r-s}}{(m+1+s) !(r-s) !}\right] \phi^{(m+1+r)}(\beta) \\
& +\int_{\alpha}^{\beta} G_{m n}(u, t) \phi^{(n)}(t) d t
\end{aligned}
$$
we get

By an easy calculation, applying (8) in $\int_{\Omega_{2}} \phi(f(y)) v(y) d \mu_{2}(y)-\int_{\Omega_{1}} \phi\left(A_{k} f(x)\right) u(x) d \mu_{1}(x)$,

$$
\begin{aligned}
& \int_{\Omega_{2}} \phi(f(y)) v(y) d \mu_{2}(y)-\int_{\Omega_{1}} \phi\left(A_{k} f(x)\right) u(x) d \mu_{1}(x) \\
& =\sum_{s=0}^{m} \frac{\phi^{(s)}(\alpha)}{s !}\left(\int_{\Omega_{2}}(f(y)-\alpha)^{s} v(y) d \mu_{2}(y)-\int_{\Omega_{1}}\left(A_{k} f(x)-\alpha\right)^{s} u(x) d \mu_{1}(x)\right)
\end{aligned}
$$




$$
\begin{aligned}
& +\sum_{r=0}^{n-m-2} \sum_{s=0}^{r} \frac{(-1)^{r-s}(\beta-\alpha)^{r-s} \phi^{(m+1+r)}(\beta)}{(m+1+s) !(r-s) !} \int_{\Omega_{2}}(f(y)-\alpha)^{m+1+s} v(y) d \mu_{2}(y) \\
& \left.\quad-\int_{\Omega_{1}}\left(A_{k} f(x)-\alpha\right)^{m+1+s} u(x) d \mu_{1}(x)\right) \\
& \left.+\int_{\alpha}^{\beta} \int_{\Omega_{2}} G_{m n}(f(y), t) v(y) d \mu_{2}(y)-\int_{\Omega_{1}} G_{m n}\left(A_{k} f(x), t\right) u(x) d \mu_{1}(x)\right) \phi^{(n)}(t) d t .
\end{aligned}
$$

Since

$$
\begin{aligned}
& \int_{\Omega_{2}} v(y) d \mu_{2}(y)-\int_{\Omega_{1}} u(x) d \mu_{1}(x) \\
& =\int_{\Omega_{2}}\left(\int_{\Omega_{1}} \frac{k(x, y)}{K(x)} u(x) d \mu_{1}(x)\right) d \mu_{2}(y)-\int_{\Omega_{1}} u(x) d \mu_{1}(x) \\
& =\int_{\Omega_{1}} \frac{u(x)}{K(x)}\left(\int_{\Omega_{2}} k(x, y) d \mu_{2}(y)\right) d \mu_{1}(x)-\int_{\Omega_{1}} u(x) d \mu_{1}(x) \\
& =\int_{\Omega_{1}} u(x) d \mu_{1}(x)-\int_{\Omega_{1}} u(x) d \mu_{1}(x)=0
\end{aligned}
$$

the summand for $s=0$ in the first sum on the right hand side is equal to zero, so (7) follows.

We continue with the following result.

Theorem 4. Let all the assumptions of Theorem 3 hold, let $\phi$ be $n$-convex on $[\alpha, \beta]$ and

$$
\int_{\Omega_{1}} G_{m n}\left(A_{k} f(x), t\right) u(x) d \mu_{1}(x) \leq \int_{\Omega_{2}} G_{m n}(f(y), t) v(y) d \mu_{2}(y), \quad t \in[\alpha, \beta] .
$$

Then

$$
\begin{aligned}
& \int_{\Omega_{2}} \phi(f(y)) v(y) d \mu_{2}(y)-\int_{\Omega_{1}} \phi\left(A_{k} f(x)\right) u(x) d \mu_{1}(x) \\
& \geq \sum_{s=1}^{m} \frac{\phi^{(s)}(\alpha)}{s !}\left(\int_{\Omega_{2}}(f(y)-\alpha)^{s} v(y) d \mu_{2}(y)-\int_{\Omega_{1}}\left(A_{k} f(x)-\alpha\right)^{s} u(x) d \mu_{1}(x)\right) \\
& +\sum_{r=0}^{n-m-2} \sum_{s=0}^{r} \frac{(-1)^{r-s}(\beta-\alpha)^{r-s} \phi^{(m+1+r)}(\beta)}{(m+1+s) !(r-s) !}\left(\int_{\Omega_{2}}(f(y)-\alpha)^{m+1+s} v(y) d \mu_{2}(y)\right. \\
& \left.-\int_{\Omega_{1}}\left(A_{k} f(x)-\alpha\right)^{m+1+s} u(x) d \mu_{1}(x)\right) .
\end{aligned}
$$

If the reverse inequality in (9) holds, then the reverse inequality in (10) holds.

Proof. We assumed that $\phi \in C^{n}([\alpha, \beta])$ is $n$-convex, so $\phi^{(n)} \geq 0$ on $[\alpha, \beta]$. We apply Theorem 3 and (10). 
Remark 2. Notice that for $n=2$ and $0 \leq m \leq 1$ the function $G_{m n}(\cdot, t), t \in[\alpha, \beta]$ is convex on $[\alpha, \beta]$. Therefore the assumption (9) is satisfied and then the inequality (10) holds. For an arbitrary $n \geq 3$ and $0 \leq m \leq 1$, we use Remark 1, i.e., we consider the following inequality:

$$
(-1)^{n-2} \frac{\partial^{2} G_{m n}(u, t)}{\partial u^{2}} \geq 0
$$

Ww conclude that the convexity of $G_{m n}(\cdot, t)$ depends of a parity of $n$. If $n$ is even, then $\frac{\partial^{2} G_{m n}(u, t)}{\partial u^{2}} \geq 0$, i.e., $G_{m n}(\cdot, t)$ is convex and assumption (9) is satisfied. Also, the inequality (10) holds. For odd $n$ we get the reverse inequality. For all other choices, the following generalization holds.

Theorem 5. Suppose that all assumptions of Theorem 1 hold. Additionally, let $n, m \in \mathbb{N}, n \geq 3$, $2 \leq m \leq n-1$ and $\phi \in C^{n}([\alpha, \beta])$ be $n$-convex.

(i) If $n-m$ is odd, then the inequality (10) holds.

(ii) If $n-m$ is even, then the reverse inequality in (10) holds.

\section{Proof.}

(i) By Remark 1, the following inequality holds

$$
(-1)^{n-m-1} \frac{\partial^{2} G_{m n}(u, t)}{\partial u^{2}} \geq 0, \quad \alpha \leq u, t \leq \beta .
$$

In case $n-m$ is odd ( $n-m-1$ is even), we have

$$
\frac{\partial^{2} G_{m n}(u, t)}{\partial u^{2}} \geq 0
$$

i.e., $G_{m n}(\cdot, t), t \in[\alpha, \beta]$, is convex on $[\alpha, \beta]$. Then by Theorem 1 we have

$$
\int_{\Omega_{1}} u(x) G_{m n}\left(A_{k} f(x), t\right) d \mu_{1}(x) \leq \int_{\Omega_{2}} v(y) G_{m n}(f(y), t) d \mu_{2}(y),
$$

i.e., the assumption (9) is satisfied. By applying Theorem 4 we get (10).

(ii) Similarly, if $n-m$ is even, then $G_{m n}(\cdot, t), t \in[\alpha, \beta]$ is concave on $[\alpha, \beta]$, so the reversed inequality in (9) holds and, hence, in (10) as well.

Theorem 6. Suppose that all assumptions of Theorem 1 hold and let $n, m \in \mathbb{N}, n \geq 2$, $0 \leq m \leq n-1, \phi \in C^{n}([\alpha, \beta])$ be $n$-convex and $F:[\alpha, \beta] \rightarrow \mathbb{R}$ be defined by

$$
\begin{aligned}
F(t) & =\sum_{s=2}^{m} \frac{\phi^{(s)}(\alpha)}{s !}(t-\alpha)^{s} \\
& +\sum_{r=0}^{n-m-2} \sum_{s=0}^{r} \frac{(-1)^{r-s}(\beta-\alpha)^{r-s}}{(m+1+s) !(r-s) !} \phi^{(m+1+r)}(\beta)(t-\alpha)^{m+1+s} .
\end{aligned}
$$

(i) If (10) holds and F is convex, then the inequality (4) holds.

(ii) If the reverse of (10) holds and $F$ is concave, then the reverse inequality (4) holds.

\section{Proof.}

(i) Let (10) holds. If $F$ is convex, then by Theorem 1 we have

$$
\int_{\Omega_{2}} v(y) F(f(y)) d \mu_{2}(y)-\int_{\Omega_{1}} u(x) F\left(A_{k} f(x)\right) d \mu_{1}(x) \geq 0
$$


which, changing the order of summation, can be written in form

$$
\begin{aligned}
& \sum_{s=1}^{m} \frac{\phi^{(s)}(\alpha)}{s !}\left(\int_{\Omega_{2}}(f(y)-\alpha)^{s} v(y) d \mu_{2}(y)-\int_{\Omega_{1}}\left(A_{k} f(x)-\alpha\right)^{s} u(x) d \mu_{1}(x)\right)+ \\
& \sum_{r=0}^{n-m-2} \sum_{s=0}^{r} \frac{(-1)^{r-s}(\beta-\alpha)^{r-s} \phi^{(m+1+r)}(\beta)}{(m+1+s) !(r-s) !} \int_{\Omega_{2}}(f(y)-\alpha)^{m+1+s} v(y) d \mu_{2}(y) \\
& \left.\quad-\int_{\Omega_{1}}\left(A_{k} f(x)-\alpha\right)^{m+1+s} u(x) d \mu_{1}(x)\right) \\
& \geq 0 .
\end{aligned}
$$

We conclude that the right-hand side of (10) is nonnegative and the inequality (4) follows.

(ii) Similar to (i) case.

Remark 3. Note that the function $t \mapsto(t-\alpha)^{p}$ is convex on $[\alpha, \beta]$ for each $p=2, \ldots, n-1$, i.e.,

$$
\int_{\Omega_{2}} v(y)(f(y)-\alpha)^{p} d \mu_{2}(y)-\int_{\Omega_{1}} u(x)\left(A_{k} f(x)-\alpha\right)^{p} d \mu_{1}(x) \geq 0,
$$

for each $p=2, \ldots, n-1$.

(i) If (10) holds, $\phi^{(s)}(\alpha) \geq 0$ for $s=0, \ldots, m$ and $(-1)^{r-s} \phi^{(m+1+r)}(\beta) \geq 0$ for $s=0, \ldots, r$ and $r=0, \ldots, n-m-2$ then the right hand side of (10) is non-negative, i.e., the inequality (4) holds.

(ii) If the reverse of (10) holds, $\phi^{(s)}(\alpha) \leq 0$ for $s=0, \ldots, m$ and $(-1)^{r-s} \phi^{(m+1+s)}(\beta) \leq 0$ for $s=0, \ldots, r$ and $r=0, \ldots, n-m-2$, then the right hand side of (10) is negative, i.e., the reverse inequality in (4) holds.

\section{Upper Bound for Generalized Hardy's Inequality}

The following estimations for Hardy's difference is given in the previous section, under special conditions in Theorem 6 and Remark 3.

$$
\begin{aligned}
& \int_{\Omega_{2}} \phi(f(y)) v(y) d \mu_{2}(y)-\int_{\Omega_{1}} \phi\left(A_{k} f(x)\right) u(x) d \mu_{1}(x) \\
& \geq \sum_{s=1}^{m} \frac{\phi^{(s)}(\alpha)}{s !}\left(\int_{\Omega_{2}}(f(y)-\alpha)^{s} v(y) d \mu_{2}(y)-\int_{\Omega_{1}}\left(A_{k} f(x)-\alpha\right)^{s} u(x) d \mu_{1}(x)\right) \\
& +\sum_{r=0}^{n-m-2} \sum_{s=0}^{r} \frac{(-1)^{r-s}(\beta-\alpha)^{r-s} \phi^{(m+1+r)}(\beta)}{(m+1+s) !(r-s) !}\left(\int_{\Omega_{2}}(f(y)-\alpha)^{m+1+s} v(y) d \mu_{2}(y)\right. \\
& \left.\quad-\int_{\Omega_{1}}\left(A_{k} f(x)-\alpha\right)^{m+1+s} u(x) d \mu_{1}(x)\right) \\
& \geq 0
\end{aligned}
$$

In this section, we present upper bounds for obtained generalization. We recall recent results related to the Chebyshev functional. For two Lebesgue integrable functions $g, h:[a, b] \rightarrow \mathbb{R}$ we consider the Chebyshev functional. 


$$
T(g, h)=\frac{1}{\beta-\alpha} \int_{\alpha}^{\beta} g(t) h(t) d t-\frac{1}{\beta-\alpha} \int_{\alpha}^{\beta} g(t) d t \cdot \frac{1}{\beta-\alpha} \int_{\alpha}^{\beta} h(t) d t .
$$

With $\|\cdot\|_{p}, 1 \leq p \leq \infty$, we denote the usual Lebesgue norms on space $L_{p}[a, b]$.

In [12] authors proved the following theorems.

Theorem 7. Let $g:[\alpha, \beta] \rightarrow \mathbb{R}$ be a Lebesque integrable function and $h:[\alpha, \beta] \rightarrow \mathbb{R}$ be an absolutely continuous function with $(\cdot-a)(b-\cdot)\left[h^{\prime}\right]^{2} \in L[\alpha, \beta]$. Then we have the inequality

$$
T(g, h) \mid \leq \frac{1}{\sqrt{2}}[T(g, g)]^{\frac{1}{2}} \frac{1}{\sqrt{\beta-\alpha}}\left(\int_{\alpha}^{\beta}(x-\alpha)(\beta-x)\left[h^{\prime}(x)\right]^{2} d x\right)^{\frac{1}{2}} .
$$

The constant $\frac{1}{\sqrt{2}}$ in (12) is the best possible.

Theorem 8. Assume that $h:[\alpha, \beta] \rightarrow \mathbb{R}$ is monotonic nondecreasing on $[\alpha, \beta]$ and $g:[\alpha, \beta] \rightarrow \mathbb{R}$ is absolutely continuous with $g^{\prime} \in L_{\infty}[\alpha, \beta]$. Then we have the inequality

$$
|T(g, h)| \leq \frac{1}{2(\beta-\alpha)} \mid\left\|g^{\prime}\right\|_{\infty} \int_{\alpha}^{\beta}(x-\alpha)(\beta-x) d h(x) .
$$

The constant $\frac{1}{2}$ in (13) is the best possible.

Under assumptions of Theorem 3 we define the function $\mathcal{L}:[\alpha, \beta] \rightarrow \mathbb{R}$ by

$$
\mathcal{L}(t)=\int_{\Omega_{2}} v(y) G_{m n}(f(y), t) d \mu_{2}(y)-\int_{\Omega_{1}} u(x) G_{m n}\left(A_{k} f(x), t\right) d \mu_{1}(x) .
$$

The Chebyshev functional is defined by

$$
T(\mathcal{L}, \mathcal{L})=\frac{1}{\beta-\alpha} \int_{\alpha}^{\beta} \mathcal{L}^{2}(t) d t-\left(\frac{1}{\beta-\alpha} \int_{\alpha}^{\beta} \mathcal{L}(t) d t\right)^{2}
$$

Theorem 9. Suppose that all the assumptions of Theorem 3 hold. Also, let $(\cdot-\alpha)(\beta-\cdot)\left(\phi^{(n+1)}\right)^{2}$ $\in L_{1}[\alpha, \beta]$ and $\mathcal{L}$ be defined as in (14). Then the following identity holds:

$$
\begin{aligned}
& \int_{\Omega_{2}} \phi(f(y)) v(y) d \mu_{2}(y)-\int_{\Omega_{1}} \phi\left(A_{k} f(x)\right) u(x) d \mu_{1}(x) \\
& =\sum_{s=1}^{m} \frac{\phi^{(s)}(\alpha)}{s !}\left(\int_{\Omega_{2}}(f(y)-\alpha)^{s} v(y) d \mu_{2}(y)-\int_{\Omega_{1}}\left(A_{k} f(x)-\alpha\right)^{s} u(x) d \mu_{1}(x)\right) \\
& +\sum_{r=0}^{n-m-2} \sum_{s=0}^{r} \frac{(-1)^{r-s}(\beta-\alpha)^{r-s} \phi^{(m+1+r)}(\beta)}{(m+1+s) !(r-s) !}\left(\int_{\Omega_{2}}(f(y)-\alpha)^{m+1+s} v(y) d \mu_{2}(y)\right. \\
& \left.-\int_{\Omega_{1}}\left(A_{k} f(x)-\alpha\right)^{m+1+s} u(x) d \mu_{1}(x)\right) \\
& +\frac{\phi^{(n-1)}(\beta)-\phi^{(n-1)}(\alpha)}{\beta-\alpha} \int_{\alpha}^{\beta} \mathcal{L}(t) d t+R(\alpha, \beta ; \phi),
\end{aligned}
$$


where the remainder $R(\alpha, \beta ; \phi)$ satisfies the estimation

$$
|R(\alpha, \beta ; \phi)| \leq \sqrt{\frac{\beta-\alpha}{2}}[T(\mathcal{L}, \mathcal{L})]^{\frac{1}{2}}\left|\int_{\alpha}^{\beta}(t-\alpha)(\beta-t)\left[\phi^{(n+1)}(t)\right]^{2} d t\right|^{\frac{1}{2}} .
$$

Proof. Applying Theorem 7 for $g \rightarrow \mathcal{L}$ and $h \rightarrow \phi^{(n)}$ we get

$$
\begin{aligned}
& \left|\frac{1}{\beta-\alpha} \int_{\alpha}^{\beta} \mathcal{L}(t) \phi^{(n)}(t) d t-\frac{1}{\beta-\alpha} \int_{\alpha}^{\beta} \mathcal{L}(t) d t \cdot \frac{1}{\beta-\alpha} \int_{\alpha}^{\beta} \phi^{(n)}(t) d t\right| \\
& \leq \frac{1}{\sqrt{2}}[T(\mathcal{L}, \mathcal{L})]^{\frac{1}{2}} \frac{1}{\sqrt{\beta-\alpha}}\left|\int_{\alpha}^{\beta}(t-\alpha)(\beta-t)\left[\phi^{(n+1)}(t)\right]^{2} d t\right|^{\frac{1}{2}} .
\end{aligned}
$$

Therefore, we have

$$
\int_{\alpha}^{\beta} \mathcal{L}(t) \phi^{(n)}(t) d t=\frac{\phi^{(n-1)}(\beta)-\phi^{(n-1)}(\alpha)}{\beta-\alpha} \int_{\alpha}^{\beta} \mathcal{L}(t) d t+R(\alpha, \beta ; \phi),
$$

where the remainder $R(\alpha, \beta ; \phi)$ satisfies the estimation. Now from the identity (7) we obtain (15).

The following Grüss type inequality also holds.

Theorem 10. Suppose that all the assumptions of Theorem 3 hold. Let $\phi^{(n+1)} \geq 0$ on $[\alpha, \beta]$ and $\mathcal{L}$ be defined as in (14). Then the identity (15) holds and the remainder $R(\phi ; a, b)$ satisfies the bound

$$
|R(\alpha, \beta ; \phi)| \leq\left\|\mathcal{L}^{\prime}\right\|_{\infty}\left\{\frac{\phi^{(n-1)}(\beta)+\phi^{(n-1)}(\alpha)}{2}-\frac{\phi^{(n-2)}(\beta)-\phi^{(n-2)}(\alpha)}{\beta-\alpha}\right\} .
$$

Proof. By applying Theorem 8 for $g \rightarrow \mathcal{L}$ and $h \rightarrow \phi^{(n)}$ we obtain

$$
\begin{aligned}
& \left|\frac{1}{\beta-\alpha} \int_{\alpha}^{\beta} \mathcal{L}(t) \phi^{(n)}(t) d t-\frac{1}{\beta-\alpha} \int_{\alpha}^{\beta} \mathcal{L}(t) d t \cdot \frac{1}{\beta-\alpha} \int_{\alpha}^{\beta} \phi^{(n)}(t) d t\right| \\
& \leq \frac{1}{2(\beta-\alpha)}\left\|\mathcal{L}^{\prime}\right\|_{\infty} \int_{\alpha}^{\beta}(t-\alpha)(\beta-t) \phi^{(n+1)}(t) d t .
\end{aligned}
$$

Since

$$
\begin{aligned}
& \int_{\alpha}^{\beta}(t-\alpha)(\beta-t) \phi^{(n+1)}(t) d t=\int_{\alpha}^{\beta}[2 t-(\alpha+\beta)] \phi^{(n)}(t) d t \\
& =(\beta-\alpha)\left[\phi^{(n-1)}(\beta)+\phi^{(n-1)}(\alpha)\right]-2\left(\phi^{(n-2)}(\beta)-\phi^{(n-2)}(\alpha)\right),
\end{aligned}
$$

using the identities (7) and (17) we deduce (16).

We continue with the following result that is an upper bound for generalized Hardy's inequality.

Theorem 11. Suppose that all the assumptions of Theorem 3 hold. Let $(p, q)$ be a pair of conjugate exponents, that is $1 \leq p, q \leq \infty, \frac{1}{p}+\frac{1}{q}=1$. Then 


$$
\begin{aligned}
& \mid \int_{\Omega_{2}} \phi(f(y)) v(y) d \mu_{2}(y)-\int_{\Omega_{1}} \phi\left(A_{k} f(x)\right) u(x) d \mu_{1}(x) \\
& -\sum_{s=1}^{m} \frac{\phi^{(s)}(\alpha)}{s !}\left(\int_{\Omega_{2}}(f(y)-\alpha)^{s} v(y) d \mu_{2}(y)-\int_{\Omega_{1}}\left(A_{k} f(x)-\alpha\right)^{s} u(x) d \mu_{1}(x)\right) \\
& -\sum_{r=0}^{n-m-2} \sum_{s=0}^{r} \frac{(-1)^{r-s}(\beta-\alpha)^{r-s} \phi^{(m+1+r)}(\beta)}{(m+1+s) !(r-s) !}\left(\int_{\Omega_{2}}(f(y)-\alpha)^{m+1+s} v(y) d \mu_{2}(y)\right. \\
& \left.\left.\quad-\int_{\Omega_{1}}\left(A_{k} f(x)-\alpha\right)^{m+1+s} u(x) d \mu_{1}(x)\right)\left.\left.\right|_{\Omega_{1}} u(x) G_{m n}\left(A_{k} f(x), t\right) d \mu_{1}(x)\right|^{q} d t\right)^{\frac{1}{q}} .
\end{aligned}
$$

The constant on the right-hand side of (18) is sharp for $1<p \leq \infty$ and the best possible for $p=1$.

Proof. We apply the Hölder inequality to the identity (7) and get

$$
\begin{aligned}
& \mid \int_{\Omega_{2}} \phi(f(y)) v(y) d \mu_{2}(y)-\int_{\Omega_{1}} \phi\left(A_{k} f(x)\right) u(x) d \mu_{1}(x) \\
& -\sum_{s=1}^{m} \frac{\phi^{(s)}(\alpha)}{s !}\left(\int_{\Omega_{2}}(f(y)-\alpha)^{s} v(y) d \mu_{2}(y)-\int_{\Omega_{1}}\left(A_{k} f(x)-\alpha\right)^{s} u(x) d \mu_{1}(x)\right) \\
& -\sum_{r=0}^{n-m-2} \sum_{s=0}^{r} \frac{(-1)^{r-s}(\beta-\alpha)^{r-s} \phi^{(m+1+r)}(\beta)}{(m+1+s) !(r-s) !}\left(\int_{\Omega_{2}}(f(y)-\alpha)^{m+1+s} v(y) d \mu_{2}(y)\right. \\
& \left.\quad-\int_{\Omega_{1}}\left(A_{k} f(x)-\alpha\right)^{m+1+s} u(x) d \mu_{1}(x)\right) \mid \\
& =\left|\int_{\alpha}^{\beta}\left(\int_{\Omega_{2}} v(y) G_{m n}(f(y), t) d \mu_{2}(y)-\int_{\Omega_{1}} u(x) G_{m n}\left(A_{k} f(x), t\right) d \mu_{1}(x)\right) \phi^{(n)}(t) d t\right| \\
& \leq|| \phi^{(n)} \| \int_{p}\left(\int_{\alpha}^{\beta}|\mathcal{F}(t)|^{q} d t\right)^{\frac{1}{q}} \mid
\end{aligned}
$$

where $\mathcal{F}(t)$ is defined as in (14).

The proof of the sharpness is analog to one in proof of Theorem 11 in [13].

We continue with a particular case of Green's function $G_{m n}(u, t)$ defined by (6). For $n=2, m=1$, we have

$$
G_{12}(u, t)= \begin{cases}u-t, & \alpha \leq t \leq u \\ 0, & u \leq t \leq \beta\end{cases}
$$

If we choose $n=2$ and $m=1$ in Theorem 11, we get the following corollary.

Corollary 1. Let $\phi \in C^{2}([\alpha, \beta])$ and $(p, q)$ be a pair of conjugate exponents, that is $1 \leq p, q \leq \infty$, $\frac{1}{p}+\frac{1}{q}=1$. Then 


$$
\begin{aligned}
& \left|\int_{\Omega_{2}} \phi(f(y)) v(y) d \mu_{2}(y)-\int_{\Omega_{1}} \phi\left(A_{k} f(x)\right) u(x) d \mu_{1}(x)\right| \\
& \leq\left\|\phi^{\prime \prime}\right\|_{p}\left(\int_{\alpha}^{\beta}\left|\int_{\Omega_{2}} v(y) G_{12}(f(y), t) d \mu_{2}(y)-\int_{\Omega_{1}} u(x) G_{12}\left(A_{k} f(x), t\right) d \mu_{1}(x)\right|^{q} d t\right)^{\frac{1}{q}} .
\end{aligned}
$$
$p=1$.

The constant on the right hand side of (21) is sharp for $1<p \leq \infty$ and the best possible for

Remark 4. If we additionally suppose that $\phi$ is convex, then the difference $\int_{\Omega_{2}} \phi(f(y)) v(y) d \mu_{2}(y)-$ $\int_{\Omega_{1}} \phi\left(A_{k} f(x)\right) u(x) d \mu_{1}(x)$ is non-negative and we have

$$
\begin{aligned}
0 & \leq \int_{\Omega_{2}} \phi(f(y)) v(y) d \mu_{2}(y)-\int_{\Omega_{1}} \phi\left(A_{k} f(x)\right) u(x) d \mu_{1}(x) \\
& \leq\left\|\phi^{\prime \prime}\right\|_{p}\left(\int_{\alpha}^{\beta}\left|\int_{\Omega_{2}} v(y) G_{12}(f(y), t) d \mu_{2}(y)-\int_{\Omega_{1}} u(x) G_{12}\left(A_{k} f(x), t\right) d \mu_{1}(x)\right|^{q} d t\right)^{\frac{1}{q}} .
\end{aligned}
$$

In sequel we consider some particular cases of this result.

Example 1. Let $\Omega_{1}=\Omega_{2}=(0, b), 0<b \leq \infty$, replace $d \mu_{1}(x)$ and $d \mu_{2}(y)$ by the Lebesque measures $d x$ and $d y$, respectively, and let $k(x, y)=0$ for $x<y \leq b$. Then $A_{k}$ coincides with the Hardy operator $H_{k}$ defined by

$$
H_{k}: H_{k} f(x):=\frac{1}{K(x)} \int_{0}^{x} f(t) k(x, t) d t,
$$

where

$$
K(x):=\int_{0}^{x} k(x, t) d t<\infty .
$$

If also $u(x)$ is replaced by $u(x) / x$ and $v(x)$ by $v(x) / x$, then

$$
\begin{aligned}
0 & \leq \int_{0}^{b} v(y) \phi(f(y)) \frac{d y}{y}-\int_{0}^{b} u(x) \phi\left(H_{k} f(x)\right) \frac{d x}{x} \\
& \leq\left\|\phi^{\prime \prime}\right\|_{p}\left(\int_{\alpha}^{\beta}\left|\int_{0}^{b} v(y) G_{12}(f(y), t) \frac{d y}{y}-\int_{0}^{b} u(x) G_{12}\left(H_{k} f(x), t\right) \frac{d x}{x}\right|^{q} d t\right)^{\frac{1}{q}} .
\end{aligned}
$$

Example 2. By arguing as in Example 1 but $\Omega_{1}=\Omega_{2}=(b, \infty), 0 \leq b<\infty$ and with kernels such that $k(x, y)=0$ for $b \leq y<x$ we obtain the following result 


$$
\begin{aligned}
0 & \leq \int_{b}^{\infty} \phi(f(y)) v(y) \frac{d y}{y}-\int_{b}^{\infty} \phi\left(H_{\bar{k}} f(x)\right) u(x) \frac{d x}{x} \\
& \leq\left\|\phi^{\prime \prime}\right\|_{p}\left(\int_{\alpha}^{\beta}\left|\int_{b}^{\infty} v(y) G_{12}(f(y), t) \frac{d y}{y}-\int_{b}^{\infty} u(x) G_{12}\left(H_{\bar{k}} f(x), t\right) \frac{d x}{x}\right|^{q} d t\right)^{\frac{1}{q}} .
\end{aligned}
$$

where the dual Hardy operator $H_{\bar{k}} f$ is defined by

$$
H_{\bar{k}} f(x):=\frac{1}{\bar{K}(x)} \int_{x}^{\infty} k(x, y) f(y) d y,
$$

where $\bar{K}(x)=\int_{x}^{\infty} k(x, y) d y<\infty$.

We continue with the following Example.

Example 3. Let $\Omega_{1}=\Omega_{2}=(0, \infty)$ and $k(x, y)=1,0 \leq y \leq x, k(x, y)=0, y>x, d \mu_{1}(x)=$ $d x, d \mu_{2}(y)=d y$ and $u(x)=\frac{1}{x}$ (so that $v(y)=\frac{1}{y}$ ) we obtain the following result

$$
\begin{aligned}
0 & \leq \int_{0}^{\infty} \phi(f(y)) \frac{d y}{y}-\int_{0}^{\infty} \phi\left(A_{k} f(x)\right) \frac{d x}{x} \\
& \leq\left\|\phi^{\prime \prime}\right\|_{p}\left(\int_{\alpha}^{\beta}\left|\int_{0}^{\infty} G_{12}(f(y), t) \frac{d y}{y}-\int_{0}^{\infty} G_{12}\left(A_{k} f(x), t\right) \frac{d x}{x}\right|^{q} d t\right)^{\frac{1}{q}}
\end{aligned}
$$

where $A_{k}$ is defined by

$$
A_{k} f(x)=\frac{1}{x} \int_{0}^{x} f(y) d y
$$

Example 4. By arguing as in Example 3 but only with $\phi(x)=x^{p}, \prod_{i=1}^{k}(p+1-i) \geq 0$ we obtain the following result

$$
\begin{aligned}
0 & \leq \int_{0}^{\infty} f^{p}(x) \frac{d x}{x}-\int_{0}^{\infty}\left(\frac{1}{x} \int_{0}^{x} f(t) d t\right)^{p} \frac{d x}{x} \\
& \leq\left\|\phi^{\prime \prime}\right\|_{p}\left(\int_{\alpha}^{\beta}\left|\int_{0}^{\infty} G_{12}(f(y), t) \frac{d y}{y}-\int_{0}^{\infty} G_{12}\left(A_{k} f(x) f(x), t\right) \frac{d x}{x}\right|^{q} d t\right)^{\frac{1}{q}}
\end{aligned}
$$

We continue with the result that involves Hardy-Hilbert's inequality. If $p>1$ and $f$ is a non-negative function such that $f \in L^{p}\left(\mathbb{R}_{+}\right)$, then

$$
\int_{0}^{\infty}\left(\int_{0}^{\infty} \frac{f(x)}{x+y} d x\right)^{p} d y \leq\left(\frac{\pi}{\sin \left(\frac{\pi}{p}\right)}\right)^{p} \int_{0}^{\infty} f^{p}(y) d y .
$$

Inequality (26) is sometimes called Hilbert's inequality even if Hilbert himself only considered the case $p=2$. 
Example 5. Let $\Omega_{1}=\Omega_{2}=(0, \infty)$, replace $d \mu_{1}(x)$ and $d \mu_{2}(y)$ by the Lebesque measures $d x$ and $d y$, respectively. Let $k(x, y)=\frac{\left(\frac{y}{x}\right)^{-1 / p}}{x+y}, p>1$ and $u(x)=\frac{1}{x}$. Then $K(x)=K=\frac{\pi}{\sin (\pi / p)}$ and $v(y)=\frac{1}{y}$. Let $\phi(u)=u^{p}, \prod_{i=1}^{k}(p-i+1) \geq 0$, replace $f(y)$ with $f(y) y^{\frac{1}{p}}$ then the following result follows

$$
\begin{aligned}
0 & \leq \int_{0}^{\infty} f^{p}(y) d y-K^{-p} \int_{0}^{\infty}\left(\int_{0}^{\infty} \frac{f(y)}{x+y} d y\right)^{p} d x \\
& \leq\left\|\phi^{\prime \prime}\right\|_{p}\left(\int_{\alpha}^{\beta}\left|\int_{0}^{\infty} G_{12}\left(f(y) y^{\frac{1}{p}}, t\right) \frac{d y}{y}-\int_{0}^{\infty} G_{12}\left(A_{k} f(x), t\right) \frac{d x}{x}\right|^{q} d t\right)^{\frac{1}{q}}
\end{aligned}
$$

where

$$
A_{k} f(x)=\frac{\sin (\pi / p)}{\pi} \int_{0}^{\infty} \frac{f(y)}{x+y} x^{\frac{1}{p}} d y .
$$

We also mention Pólya-Knopp's inequality,

$$
\int_{0}^{\infty} \exp \left(\frac{1}{x} \int_{0}^{x} \ln f(t) d t\right) d x<e \int_{0}^{\infty} f(x) d x,
$$

for positive functions $f \in L^{1}\left(\mathbb{R}_{+}\right)$. Pólya-Knopp's inequality may be considered as a limiting case of Hardy's inequality since (27) can be obtained from (5) by rewriting it with the function $f$ replaced with $f^{\frac{1}{p}}$ and then by letting $p \rightarrow \infty$.

Example 6. By applying (22) with $\phi(x)=e^{x}$, and $f$ replaced by $\ln f^{p}, p>0$ we obtain that

$$
\begin{aligned}
0 & \leq \int_{\Omega_{2}} f^{p}(y) v(y) d \mu_{2}(y)-\int_{\Omega_{1}}\left[\exp \left(\frac{1}{K(x)} \int_{\Omega_{2}} k(x, y) \ln f(y) d \mu_{2}(y)\right)\right]^{p} u(x) d \mu_{1}(x) \\
& \leq\left\|\phi^{\prime \prime}\right\|_{p}\left(\int_{\alpha}^{\beta}\left|\int_{\Omega_{2}} v(y) G_{12}\left(\ln f^{p}(y), t\right) d \mu_{2}(y)-\int_{\Omega_{1}} u(x) G_{12}\left(A_{k} f(x), t\right) d \mu_{1}(x)\right|^{q} d t\right)^{\frac{1}{q}}
\end{aligned}
$$

where $k(x, y), K(x), u(x)$ and $v(y)$ are defined as in Theorem 1 and

$$
A_{k} f(x)=\frac{p}{K(x)} \int_{\Omega_{2}} k(x, y) \ln f(y) d \mu_{2}(y)
$$

At the end, we give interesting application.

Using (10), under the assumptions of Theorem 4, we define the linear functional $A: C^{n}([\alpha, \beta]) \rightarrow \mathbb{R}$ by 


$$
\begin{aligned}
A(\phi) & =\int_{\Omega_{2}} \phi(f(y)) v(y) d \mu_{2}(y)-\int_{\Omega_{1}} \phi\left(A_{k} f(x)\right) u(x) d \mu_{1}(x) \\
& -\sum_{s=1}^{m} \frac{\phi^{(s)}(\alpha)}{s !}\left(\int_{\Omega_{2}}(f(y)-\alpha)^{s} v(y) d \mu_{2}(y)-\int_{\Omega_{1}}\left(A_{k} f(x)-\alpha\right)^{s} u(x) d \mu_{1}(x)\right) \\
& -\sum_{r=0}^{n-m-2} \sum_{s=0}^{r} \frac{(-1)^{r-s}(\beta-\alpha)^{r-s} \phi^{(m+1+r)}(\beta)}{(m+1+s) !(r-s) !}\left(\int_{\Omega_{2}}(f(y)-\alpha)^{m+1+s} v(y) d \mu_{2}(y)\right. \\
& \left.-\int_{\Omega_{1}}\left(A_{k} f(x)-\alpha\right)^{m+1+s} u(x) d \mu_{1}(x)\right) .
\end{aligned}
$$

If $\phi \in C^{n}([\alpha, \beta])$ is $n$-convex, then $A(\phi) \geq 0$ by Theorem 4 . Using the positivity and the linearity of functional $A$ we can get corresponding mean-value theorems. We may also obtain new classes of exponentially convex functions and get new means of the Cauchy type applying the same method as given in [14-21].

Author Contributions: Conceptualization, M.P.; Data curation, K.K.H.; Formal analysis, K.K.H. and D.P.; Investigation, K.K.H., D.P. and M.P.; Methodology, J.P. and D.P.; Supervision, J.P. All authors have read and agreed to the published version of the manuscript.

Funding: This research received no external funding.

Conflicts of Interest: The authors declare no conflict of interest.

\section{References}

1. Krulić, K.; Pečarić, J.; Persson, L.-E. Some new Hardy-type inequalities with general kernels. Math. Inequal. Appl. 2009, 12, 473-485.

2. Krulić Himmelreich, K.; Pečarić, J.; Pokaz, D. Inequalities of Hardy and Jensen; Element: Zagreb, Croatia, 2013.

3. Hardy, G.H. Notes on some points in the integral calculus LX: An inequality between integrals. Messenger Math. 1925, 54, 150-156.

4. Kufner, A.; Maligranda, L.; Persson, L.-E. The prehistory of the Hardy inequality. Am. Math. Mon. 2006, 113, 715-732. [CrossRef]

5. Kufner, A.; Maligranda, L.; Persson, L.-E. The Hardy Inequality. About Its History and Some Related Results; Vydavatelsky Servis Publishing House: Pilsen, Czech Republic, 2007.

6. Kuczma, M. An Introduction to the Theory of Functional Equations and Inequalities; Birkhauser: Basel, Switzerland; Boston, MA, USA; Berlin, Germany, 2009.

7. Pečarić, J.E.; Proschan, F.; Tong, Y.L. Convex Functions, Partial Orderings and Statistical Applications; Academic Press: San Diego, CA, USA, 1992.

8. Whittaker, J.M. Interpolation Function Theory; Cambridge University Press: Cambridge, UK, 1935.

9. Gontscharoff, V.L. Theory of Interpolation and Approximation of Functions; Gostekhizdat: Moscow, Russia, 1954.

10. Davis, P.J. Interpolation and Approximation; Blaisdell: Boston, MA, USA, 1961.

11. Agarwal, R.P.; Wong, P.J.Y. Error Inequalities in Polynomial Interpolation and Their Applications; Kluwer Academic Publishers: Dordrecht, The Netherlands, 1993.

12. Cerone, P.; Dragomir, S.S. Some new Ostrowski-type bounds for the Chebyshev functional and applications. J. Math. Inequal. 2014, 8, 159-170. [CrossRef]

13. Bradanović, S.I.; Pečarić, J. Generalizations of Sherman's inequality by Taylor's formula. J. Inequalities Spec. Funct. 2017, 8, 18-30.

14. Agarwal, R.P.; Gala, S.; Ragusa, M.A. A regulatory criterion in weak spaces to Boussinesq equations. Mathematics 2020, 8, 920 [CrossRef]

15. Agarwal, R.P.; Ivelić Bradanović, S.; Pečarić, J. Generalizations of Sherman's inequality by Lidstone's interpolationg polynomial. J. Inequal. Appl. 2016, 6, 1-18.

16. Iqbal, W.; Noor, M.A.; Noor, K.I.; Safdar, F. Generalized Petrovicś inequalities for coordinated exponentially m-convex fuctions. Int. J. Anal. Appl. 2021, 19, 296-318.

17. Jakšetić, J.; Pečarić, J. Exponetial convexity method. J. Convex Anal. 2013, 20, 181-197.

18. Krulić Himmelreich, K.; Pečarić, J. Some new Hardy-type inequalities with general kernels II. Math. Inequal. Appl. 2016, 19, 73-84.

19. Krulić Himmelreich, K.; Pečarić, J.; Praljak, M. Generalizations of Hardy type inequalities via new Green functions. submitted. 
20. Krulić Himmelreich, K.Generalizations of Hardy type inequalities by Taylor's formula. submitted.

21. Krulić Himmelreich, K.; Pečarić, J.; Praljak, M. Hardy type inequalities involving Lidstone interpolation polynomials. submitted. 